\section{An Agent-Based Model for Exploring Wolf Recolonization in Austria}

GI_Forum 2016, Vol.2

Page: 3-19

Full Paper

Corresponding Author:

scrook@sdsu.edu

DOI: 10.1553/giscience2016_02_s3

\author{
Stephen E. S. Crook' and Gernot Paulus² \\ 1 San Diego State University, USA \\ 2 Carinthia University of Applied Sciences, Villach, Austria
}

\begin{abstract}
Austria is one of the few countries in Europe that has not been recolonized by stable populations of wolves, yet many dispersing individuals have been observed. Understanding spatial and temporal patterns of recolonization can help prepare management agencies for conflict that may arise and allow for adaptive management, yet characterizations of the recolonization process are lacking in most areas where it is occurring. Here, a geospatial application of an agent-based model was explored as a potential tool in characterizing spatial and temporal patterns of wolf recolonization in Austria. Sub-models for wolf appearance in Austria, dispersal through a habitat-suitability model, mating, pack formation and death were developed in the Agent Analyst programming environment and parameterized with literature-derived values. Model outputs included total wolf numbers, wolf presence locations, and the number and location of packs formed. Throughout model runs, wolf presence locations were predictably focused near known neighbouring populations; yet different parameterizations resulted in varied larger-scale movement patterns. About half of all runs resulted in pack formation, predominantly near the Slovenian and Italian borders, indicating that it is possible for the model to predict recolonization. Although the approach has high uncertainty, it can lend insight into recolonization and can be refined through the collection of more empirical data and the application of further research into wolf decision-making.
\end{abstract}

\title{
Keywords:
}

agent-based modelling, conservation GIS, environmental management, wolf recolonization

\section{Indroduction}

The combination of agent-based modelling (ABM) and geographic information systems (GIS) allows the exploration of complex real-world systems in a novel, process-based manner (Macal \& North, 2009). Here, an ABM is developed based on a timely issue in Austria with marked potential for human conflict: the recolonization by wolves of areas where they have been missing for over a hundred years. Modelling the processes of wolf movement in Austria can give insight into priority areas for wildlife managers, allow 
exploration of scenarios for the recolonization process, and create a framework that can be refined and expanded as more becomes known about the recolonization process.

In the middle of the twentieth century, predominantly negative attitudes towards wolves began to change positively in most developed countries, resulting in support for the establishment of laws for their protection (Zeiler et al., 1999). In Europe, these changes have resulted in the expansion of wolf ranges into countries where they were previously absent. However, there are management and policy challenges inherent in negotiating the return of wolves in human-dominated landscapes. Range expansion often occurs in areas of livestock production, causing conflict between government agencies, conservationists, and residents fearing for their livelihoods (Enserink \& Vogel, 2006). Many consider wolves a risk to human safety and as competitors for finite prey populations that hunters value. These issues have resulted in humans continuing as the primary threat to wolves: one study tracking dispersing wolves in Spain identified illegal hunting and automobile strikes as the cause of $>90 \%$ of wolf deaths (Blanco \& Cortés, 2007). Predicting the dynamics of recolonization can help address these issues, allowing management agencies to prepare communities to deal with potential conflict (Marucco \& McIntire, 2010).

\section{Agent-based modelling for carnivore dispersal}

Agent-based modelling (ABM), which consists of modelling individual autonomous agents' interactions through space and time based on programmed parameters and rule sets, has been identified as having potential in informing the management of carnivores (Chapron \& Arlettaz, 2006). ABMs consist of representations of the individual components of the parts of the system that interact over time based on goals, rule sets, feedback and learning, which are programmed into the model using an object-oriented programming language (Parker et al., 2003). ABMs have been shown to reliably represent behavioural ecology through the modelling of habitat-selection and movement (Pitt et al., 2003; Rabinowitz \& Zeller, 2010). After models are developed, they can be used to analyse policies that could affect the trajectory of the system. For example, Marucco and MacIntyre (2010) use an ABM to predict the impact of wolf recolonization on livestock in the French-Italian border region by incorporating data on dispersal, habitat selection, reproduction and mortality.

\section{Wolves in Austria}

Austria was historically home to healthy wolf populations, yet by 1882 all breeding populations had been hunted, poisoned and trapped to local extinction (Dungler, 2008). During the 20th century, fewer than two dozen wolves were observed, all of which were thought to have immigrated from neighbouring countries (Schafer, 2012). In the second half of the century, wolf populations across Europe expanded, and there have been increasing numbers of encounters with wolves in Austria since the 1990s (Salvatori \& Linnell, 2005). It is estimated that between 2009 and 2011 there were between two and eight wolves at any given time within Austria (Schafer, 2012). Genetic evidence has identified the source populations of individuals as Balkan, Carpathian or Appenine (Schafer, 2012). Despite an increase in individuals, pack establishment has not occurred in Austria. 


\section{Research goals}

Currently, there is little research on the process of wolf recolonization in Austria. The objective of this research is to assess the potential of creating an ABM using the patternoriented modelling (POM) approach to derive insight into the spatial and temporal patterns of wolf recolonization in Austria, using primarily literature-derived parameters for wolf presence, movement and behaviour (Railsback \& Grimm, 2012). With POM, the goal of modelling is to distill the entities, state variables and processes that represent the system of interest (Railsback \& Grimm, 2012). The "pattern" in POM can refer to anything beyond random variation, and here refers to patterns derived from the literature that describe the natural processes underpinning each sub-model and the recolonization process as a whole. This research includes the first two steps outlined by Wiegand et al. (2004) as essential for population models in fragmented landscapes: building an ABM framework of the key processes of population dynamics and describing the response of individuals to landscape structure and the use of a habitat map that represents the spatial structure of the landscape. However, due to the lack of independent, spatially-explicit population data to use for adjusting unknown parameters, the approach here can be described as exploratory, where "modelers must make guesses at details and mechanisms" and use a series of experiments with different assumptions (in this case scenarios) to reveal a range of outputs (Bankes, 1993). While acknowledging the high uncertainty inherent in these approaches, this model aims to investigate the number of packs formed, the number of wolves present, and the average number of wolves existing over a five-year period for a set of plausible parameterizations. Additionally, the spatial configuration of dispersal and new pack formation in Austria can be modelled and scenarios explored (reintroduction, changes in human attitudes, etc.). Ultimately, by extending and properly validating this model, it could be used to inform management planning and identify new research topics concerning wolf recolonization.

\section{ODD Protocol}

The description of the methods used to construct the model follows the ODD (overview, design concepts, details) protocol that has become the standard for describing ABMs (Grimm et al., 2010). Model implementation was carried out in Agent Analyst, an open source extension to ArcGIS that integrates ArcGIS with Repast - the former providing an environment for data creation, data management, visual display of output, and GIS analysis, and the latter allowing rule set programming and scheduling of sub-models (Johnston et al., 2013). 


\section{Overview}

\section{Purpose}

The purpose of the model is to simulate spatial and temporal patterns of wolf dispersal in Austria. Once patterns have been established, the model is intended to assess the potential of exploring different scenarios resulting in wolf recolonization via pack formation.

\section{Entities, state variables and scales}

The model contains three entities: individual wolf agents, packs formed by multiple wolf agents, and patches of habitat over which processes operate. Wolf agents are described by the following state variables: name/id, sex, $\mathrm{x}$ and $\mathrm{y}$ coordinates, whether alive, whether mated, and whether they have formed a pack. Packs are formed as the outcome of a number of different decision- and proximity-based rules for wolf agents that result in the establishment of a two-dimensional area that can be written to a raster file.

The spatial extent of the model is the entirety of Austria, along with a buffer of $30 \mathrm{~km}$ beyond the national borders. Within the model, individual wolves can travel outside this area, at which point model rule sets determine whether they return to the study area or leave permanently (simulating outmigration). Each model step represents two weeks. For the movement sub-model, there are hourly sub-steps in which the wolves decide on a destination. The model is designed to be run at moderate temporal scales and a five-year time period is demonstrated.

\section{Process Overview and Scheduling}

Scheduling is illustrated in Figure 1. In the initialization step, rasters are loaded into the model and wolves are placed according to the number specified in the model parameters. In each subsequent step, a number of actions take place for each wolf. First, new wolves appear in the model environment in the appearance sub-model. Then, the move sub-model runs, in which wolf dispersal occurs at hourly sub-steps. In the mate sub-model, wolves search nearby for a mate. If there are wolves who have formed a pair, the establish pack sub-model determines whether there is sufficient quality habitat to support a pack. Finally, the die submodel results in wolves dying based on mortality probabilities, or on their leaving the study area. 


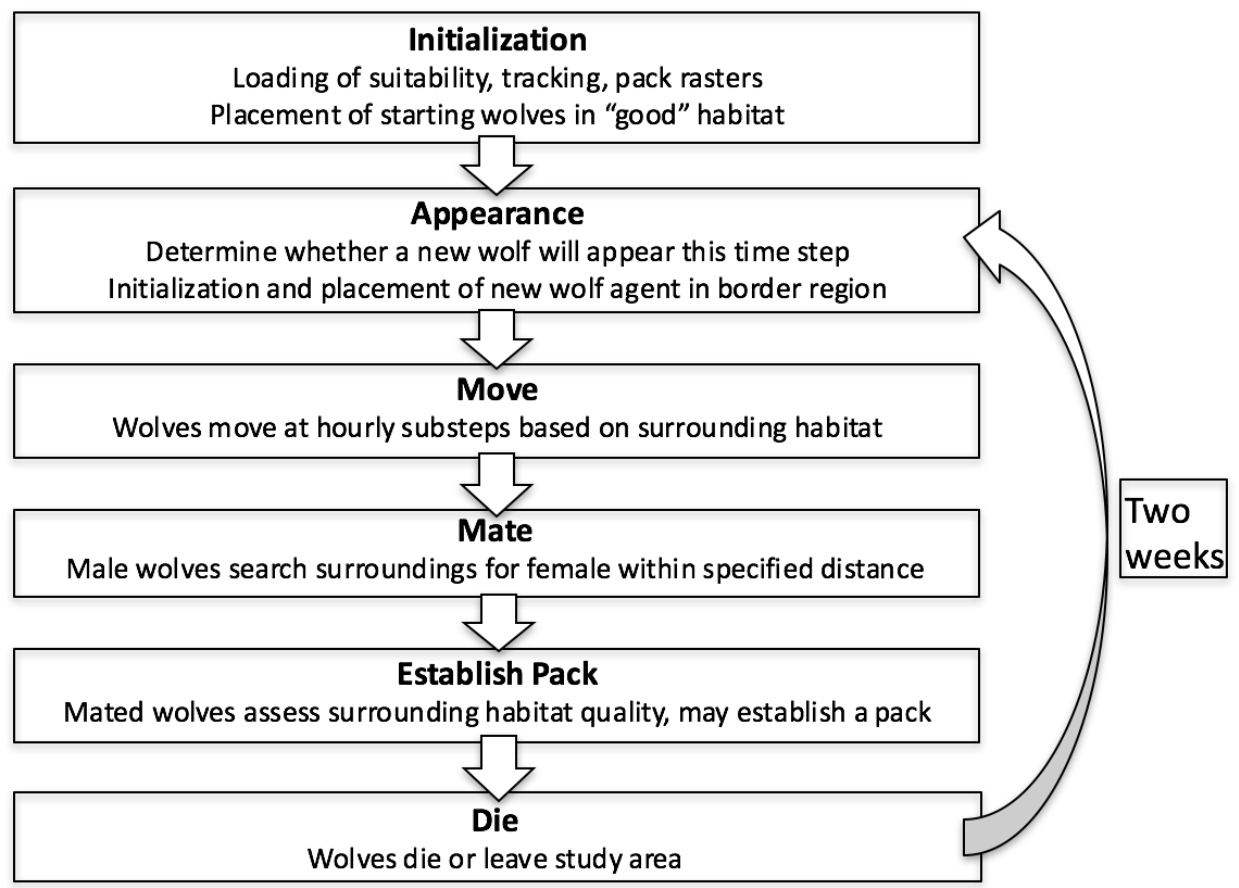

Figure 1: Process overview and scheduling

\section{Design Concepts}

The model includes the concept of emergence by capturing the system-level phenomenon of pack formation through the movements and decisions of individuals. The objectives of wolf agents are to mate and establish a pack.

The model includes sensing in three ways. First, wolves are able to sense the land cover type of the surrounding environment when they are considering whether or not to move to a given cell. Secondly, male wolves sense female wolves around themselves to find potential mates. Finally, wolves that have found a mate sense the surrounding environment when they consider the availability of good habitat for pack formation.

Wolves may interact with each other twice during a time step. First, wolves of the opposite sex are considered potential mates based on proximity, and the wolves may mate based on specified probabilities. They may further interact by forming a pack after assessing the surrounding habitat.

Stochasticity exists in the establishment of the range of possibilities of different model scenarios and movement decisions. The distance wolves travel in a given two-week period is the sum of the distances randomly drawn hourly from a normal distribution based on mean hourly wolf movement. The decision of whether to move to a different habitat is based on a random draw. Mortality at each step is determined by probabilities specified in model 
parameters. Furthermore, the decision to mate upon meeting a wolf of the opposite sex is assigned a probability.

During model runs, the spatial distribution of wolves and packs at each step can be observed. The number of packs formed and the average number of wolves alive are reported at each time step over the five-year period. Additionally, the locations where wolves exist following each time step and the locations of all of the packs formed over several model runs are written to raster files.

\section{Details}

\section{Initialization and input data}

At the initialization step, a basic habitat suitability model derived from EU Corine land cover datasets $(100 \mathrm{~m}$ resolution) is loaded into the model (Figures 2 and 3). In the habitat model, the 44 Corine classes were reclassified as low-, medium- or high-suitability as wolf habitat, representing, respectively, land cover types a wolf might avoid, consider suitable for transit, or consider suitable as core habitat. Artificial surfaces, urban areas, wetlands, water bodies and some of the "forests and seminatural areas" (glaciers and perpetual snow) were classified as low-suitability habitat. Agricultural areas made up the medium-suitability class. Most forests and semi-natural area subgroups were classified as high-suitability habitat.

After loading the raster basemap into the habitat suitability model, wolves considered to be in the study area already are placed randomly in good habitat according to the number specified in the model's parameters.

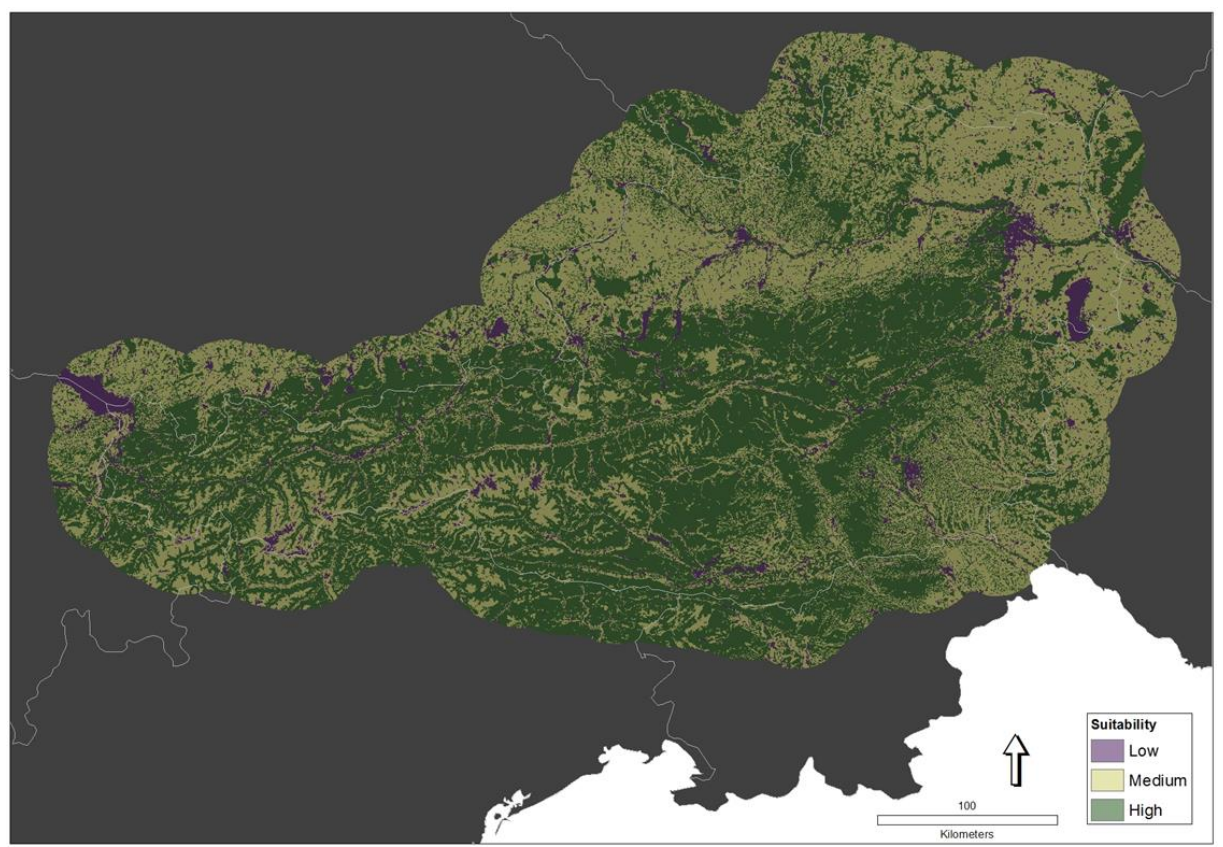

Figure 2: Habitat suitability map of study area, including the border of Austria with a $30 \mathrm{~km}$ buffer. 


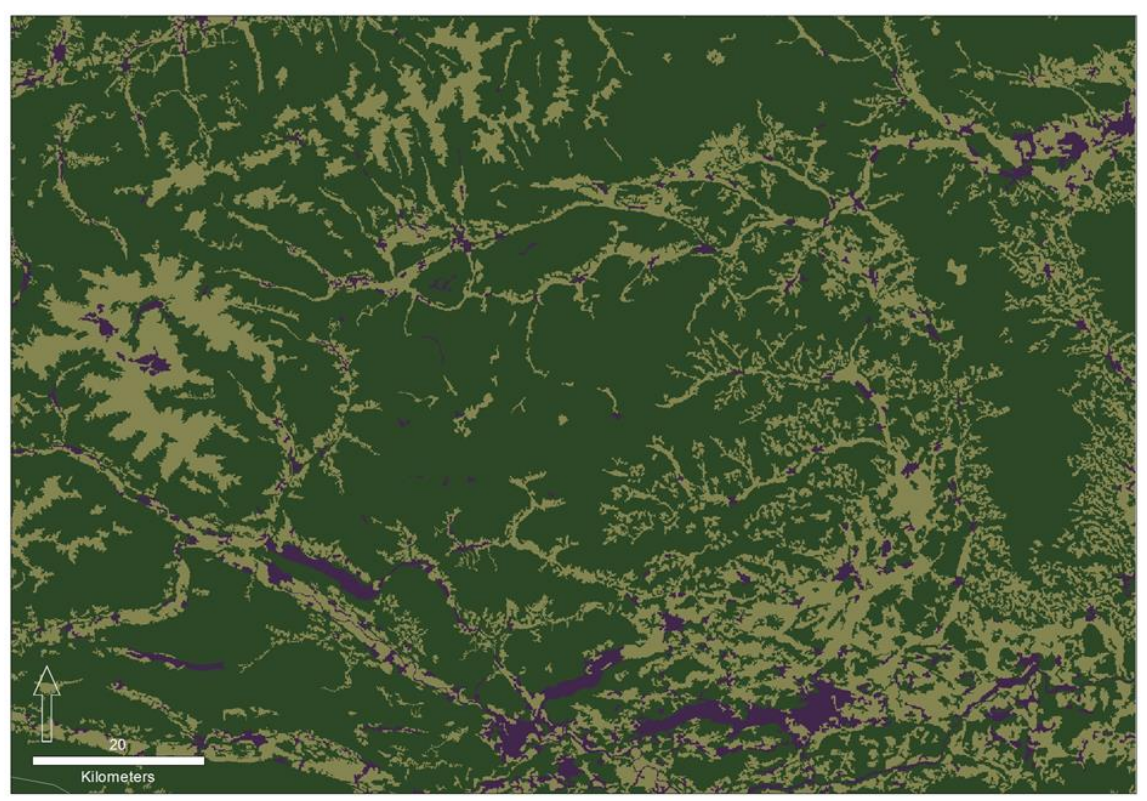

Figure 3: Habitat suitability: larger-scale map of the suitability surface in Carinthia.

\section{Sub-models}

The following sub-models run at each time step: appear, move, mate, establish pack and die. The appear sub-model runs at both the model and individual-agent levels. At the model level, the parameter for the annual rate of appearance of a new wolf is converted to a biweekly probability. A random decimal is drawn at each time step, and if that number is smaller than the biweekly probability, a wolf is created. While the model-level code indicates that a new wolf will be created, the individual-level code activates a wolf. Next, a random draw determines whether the agent comes from the Carpathian, Alpine or Dinaric population according to probabilities assigned as parameters. A wolf's sex is set by a random draw that is compared to the input parameter for the probability of new wolves being female. Finally, the new wolf is given random coordinates within the start box of the corresponding source population (Figure 4). 


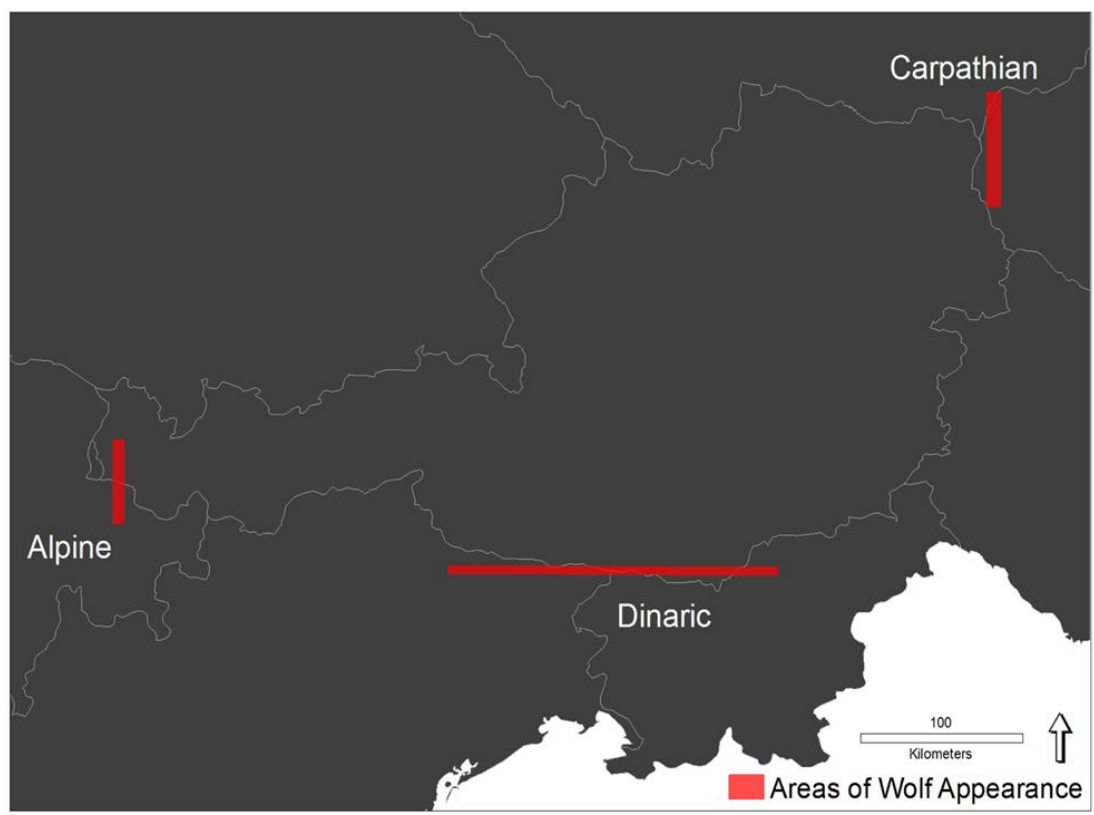

Figure 4: Start boxes for each source population (Derived from Schafer, 2012).

The move sub-model is run for living wolves that have not established a pack. Wolves move hourly, based on average movement distances and surrounding habitat types. Parameters for the daily average and standard deviations for movement are converted to hourly distances. For 336 sub-steps, $\mathrm{x}$ and $\mathrm{y}$ distances for movement are randomly drawn from normal distribution of hourly movement mean and standard deviation variables. Direction for movement (whether coordinates are positive or negative) is chosen randomly, and together with the random drawing of $\mathrm{x}$ and $\mathrm{y}$ distances, this results in the selection of a potential movement cell. However, wolves only move after considering habitat suitability, undertaking a habitat-dependent walk (similar to a habitat-dependent correlated walk; McLane et al., 2011). At each hourly sub-step, the wolf agent checks the suitability of the proposed cell, and the following rules are applied: if cell suitability is low, medium or high, the wolf agent has a $5 \%, 75 \%$ or $95 \%$ chance of moving. If the cell is out of bounds, the wolf has a $70 \%$ chance of moving, and may leave the study area permanently (see the die sub-model).

The mate sub-model allows wolves to detect other wolves of the opposite sex within a specified radius. Living male wolves that have not found a mate calculate the distance to living female wolves. If the distance between them is less than the parameter value for the maximum distance for detecting a mate, a random number draw takes place that determines whether they mate based on the mating probability parameter.

The establish pack sub-model is run by male wolves that have a mate and have not established a pack. The wolf assesses surrounding territory and establishes a pack if sufficient good habitat is found. First, the bounds of the potential pack area are calculated by converting the wolf's location as map coordinates into location on the raster grid. Bounding cells define the potential pack box based on the pack area parameter. All cells of good 
habitat within the box are summed and the wolf establishes a pack if enough good habitat is found.

The die sub-model is run by each living wolf agent. Annual mortality rate is converted to a biweekly probability and a random decimal between 0 and 1 is drawn for each living wolf. If the random number is less than the mortality probability, the wolf dies. Likewise, wolves finishing even one step outside the study area have a 30\% chance of "leaving" the study area.

\section{Model parameters and calibration}

The model was parameterized using a range of literature values and the pattern-oriented modelling approach (Table 1). Value ranges were found for average movement distance, average mortality, the distance over which wolves can detect potential mates, the area required for a pack, and the number of wolves already in Austria. Parameters developed iteratively from qualitative patterns gleaned from a general literature review or through preliminary model runs included the rate of new wolf appearance, the amount of good habitat required for a pack to form, and mating probability.

Table 1: Start parameters

\begin{tabular}{|lcll|}
\hline Parameter & Estimate Range & Units & Source \\
Mortality rate & $0.44-0.76$ & Annual death rate & Blanco and Cortes (2007), Marucco and Mclntire (2010) \\
New wolf rate & $2-4$ & Wolves per year & $\mathrm{n} / \mathrm{a}$ \\
Starting wolves & $2-8$ & Wolves & Schafer (2012) \\
Dispersal distance mean & $22.8-27.4$ & $\mathrm{~km} / \mathrm{day}$ ( $\mathrm{x}$ and y) & Jedrezejewski et al. (2001), Ciucci et al. (1997) \\
Dispersal distance std deviation & $\sim 25$ & $\mathrm{~km} / \mathrm{day}$ (x and y) & Mech (1970) \\
Mate search radius & $20.7-39.2$ & $\mathrm{~km}$ & Hurford et al. (2006) \\
Mate probability & 1 & unitless & Hurford et al. (2006) \\
Pack area & $173-294$ & $\mathrm{~km}{ }^{2}$ & Okarma et al. (1998) \\
\% Good habitat for pack formation & - & $\mathrm{good}$ habitat \% & \\
Probability female & 0.25 & & Rauer (personal communication, 2015) \\
Probability Dinaric & 0.50 & & Rauer (personal communication, 2015) \\
Probability Carpathian & 0.25 & & Rauer (personal communication, 2015) \\
Probability Alpine & 0.25 & & Rauer (personal communication, 2015) \\
\hline
\end{tabular}

Several scenarios using the literature-derived parameters that may represent the real-world system were run ten times each to demonstrate a range of model outputs (Table 2). The first scenario (S1) used values that would result in lower probabilities of wolf recolonization. The second scenario (S2) used values that would result in higher probabilities of wolf recolonization. The third scenario (S3) used intermediate values that would result in moderate probabilities of wolf recolonization. A further scenario (S4) assumed a lower mortality rate and higher new wolf rate to simulate improved human attitudes towards wolves and/or friendlier policies. Further scenarios of interest could include the exploration of the reintroduction of wolves (as demonstrated in Yellowstone National Park; Ripple \& Beschta, 2003) by placing wolves in good habitat, or by further increasing the new wolf rate to simulate population growth in the source populations surrounding the study area. 
Table 2: Start parameters for models runs

\begin{tabular}{|lccccc|}
\hline Parameter & $\begin{array}{c}\text { Model \#1 } \\
\text { (Low likelihood) }\end{array}$ & $\begin{array}{c}\text { Model \#2 } \\
\text { (High likelihood) }\end{array}$ & $\begin{array}{c}\text { Model \#3 } \\
\text { (Medium likelihood) }\end{array}$ & $\begin{array}{c}\text { Model \#4 } \\
\text { (Scenario) }\end{array}$ & Units \\
mortRate & 0.76 & 0.44 & 0.60 & 0.33 & Death rate per year \\
newWolfRate & 2 & 3 & 3 & 4 & Wolves per year \\
startingWolvesInAustria & 2 & 8 & 5 & 5 & Wolves \\
meanDailyDispersal & 16.12 & 19.37 & 17.75 & 12.00 & $\mathrm{~km} /$ day (x and y) \\
sdDailyDispersal & 16 & 19 & 17.50 & 12.00 & $\mathrm{~km} /$ day (x and y) \\
mateSearchRadius & 20.7 & 39.2 & 30.2 & 30.2 & $\mathrm{~km}$ \\
mateProbability & 1 & 1 & 1 & 1 & unitless \\
packArea & 234 & 173 & 204 & 204 & $\mathrm{~km}$ \\
percentGoodHabitatForPack & 0.85 & 0.75 & 0.80 & 0.80 & percent good habitat \\
probFemale & 0.25 & 0.25 & 0.25 & 0.25 & 0.50 \\
probDinaric & 0.50 & 0.50 & 0.50 & 0.25 & \\
probCarpathian & 0.25 & 0.25 & 0.25 & 0.25 & \\
probAlpine & 0.25 & 0.25 & & &
\end{tabular}

\section{Model Validation}

As an exploratory model, this ABM was not rigorously validated based on quantitative data. In the theory development stage of POM, the goal is to determine how well an ABM reproduces observed patterns from a variety of sources, including the literature, empirical data if available, and existing theory (Railsback \& Grimm, 2012). Following these principles, model results were qualitatively compared with how well they fitted overall patterns of wolf movement and recolonization processes discussed in the literature. As further guidance, model development and preliminary assessment were driven by local expert estimates of the approximate number of wolves that have been noted in Austria: an estimated average of 5 wolves at any given time, and 17-18 wolves in total over the last five years (Rauer, personal communication, 2015).

\section{Results}

\section{Wolf survivorship}

The model outputs included the total number of wolf agents that were alive over a model run and the average number of wolf agents alive at a given step (Figure 5). The total number existing over an entire model runs ranged from 8 to 27 in scenarios S1-S3, with the averages being 11.6, 21.7 and 17.4, respectively. The average number alive at a given time step ranged from .62 to 2.81 in models S1-S3. Scenario S4 had higher total numbers of wolf agents and a higher average number of wolf agents $(23.5,5.38)$. 


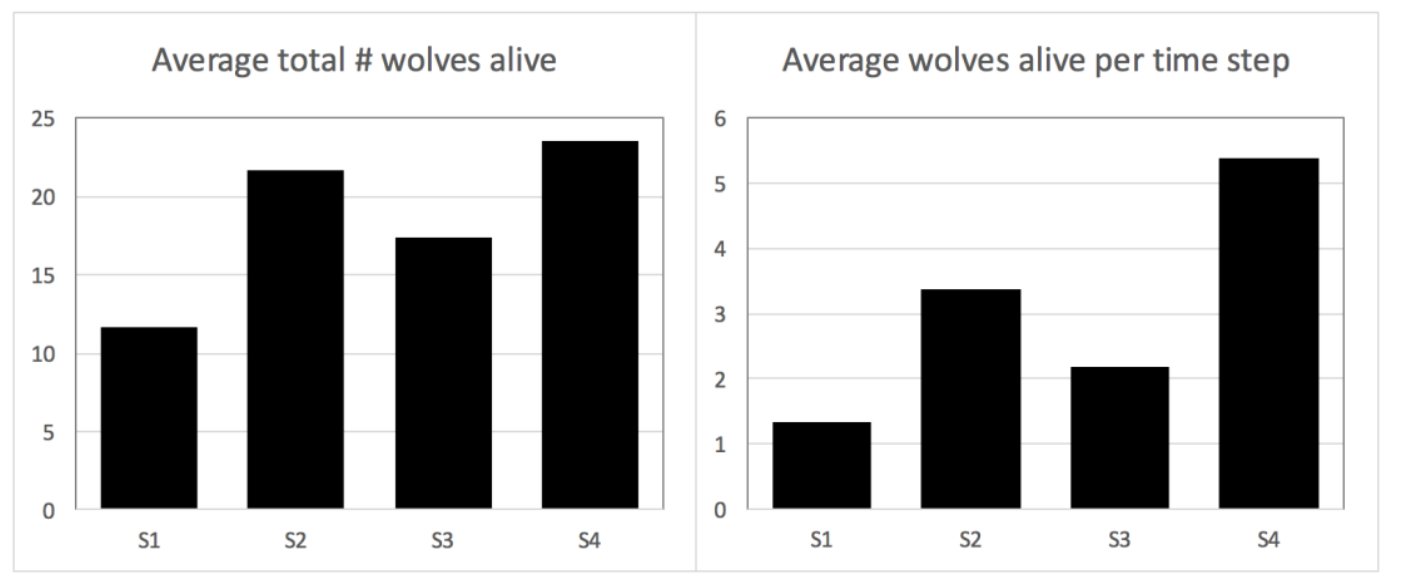

Figure 5: Total number of wolf agents and average population (10 runs)

\section{Wolf movement}

At each step, wolf agent location was documented by changing the value of the underlying cell in the tracking raster. Aggregate locations of each wolf at each time step over the ten runs of each model were visualized using kernel density estimation (KDE), indicating hotspots of wolf presence over the course of model runs (Figure 6). All models show a similar primary pattern: well-defined hotspots near wolf start locations, and decreasing presence densities away from these areas.
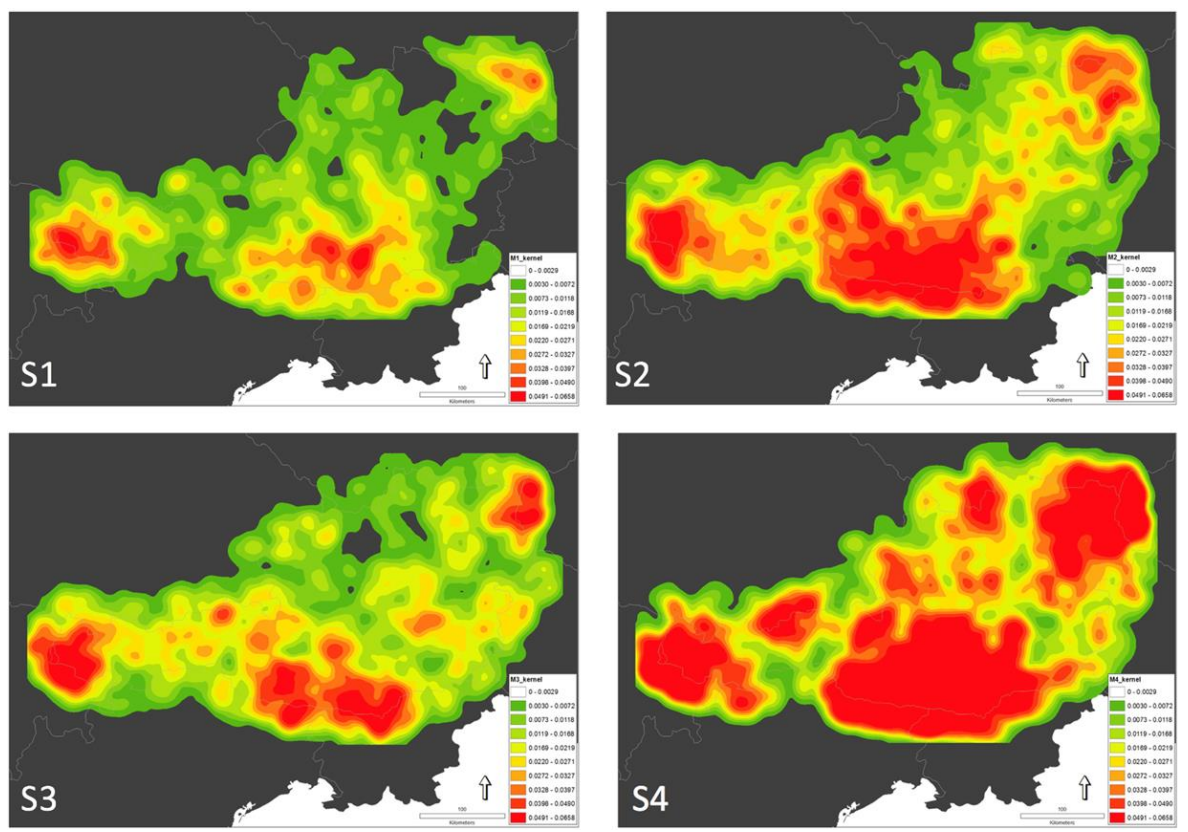

Figure 6: Kernel Density Estimation of wolf presence locations for each scenario. 
Other patterns seen in these figures show potential for the identification of areas of wolf habitat and potential movement corridors. S1 appears to have better connectivity between the Dinaric and Alpine start areas than the Dinaric and Carpathian. S2 shows corridor patterns between start areas in addition to defined hotspots. Specifically, a corridor toward the northwest part of the Dinaric start area appears well defined. S3 has more clustered hotspots than apparent corridors: one half way between the Dinaric and Carpathian start areas, and one between the Alpine and Dinaric start areas. S4 has two well-defined hotspots: in the north central part of the study area, and between the Alpine and Dinaric start areas.

\section{Pack formation}

Individual model runs over the study period resulted in a maximum of three packs being formed (Table 3). No packs were formed with S1, zero to three packs for S2, and zero to two packs for S3 and S4. The average number of packs formed was 1.20 for S2, .50 for S3, and 1.10 for S4. Data about the origin of wolf agents that formed packs was recorded during each model run. In all cases, wolf agents mated with a member of their own source population or a wolf present upon model initialization, meaning no mixing of populations was observed.

Table 3: Number of packs formed for each model run

\begin{tabular}{|lcccc|}
\hline & S1 & S2 & S3 & S4 \\
Run 1 & 0 & 1 & 0 & 1 \\
Run 2 & 0 & 1 & 0 & 0 \\
Run 3 & 0 & 1 & 0 & 0 \\
Run 4 & 0 & 1 & 1 & 1 \\
Run 5 & 0 & 2 & 2 & 2 \\
Run 6 & 0 & 0 & 0 & 2 \\
Run 7 & 0 & 3 & 0 & 1 \\
Run 8 & 0 & 1 & 1 & 2 \\
Run 9 & 0 & 1 & 0 & 0 \\
Run 10 & 0 & 1 & 1 & 2 \\
Average & 0.00 & 1.20 & 0.50 & 1.10 \\
\hline
\end{tabular}

\section{Pack locations}

Locations of pack formation were written to the "packs" raster layer for each of the ten runs for a given model, resulting in a map showing all areas where recolonization occurred. In the ten runs of S4 (Figure 7), most packs formed near where Austria, Slovenia and Italy meet. Packs also formed in areas that have large amounts of good habitat to the north and east of the Dinaric start box. These packs are the result of the meeting of two wolves placed upon model initialization or due to a Dinaric wolf agent meeting a wolf placed upon model initialization. 


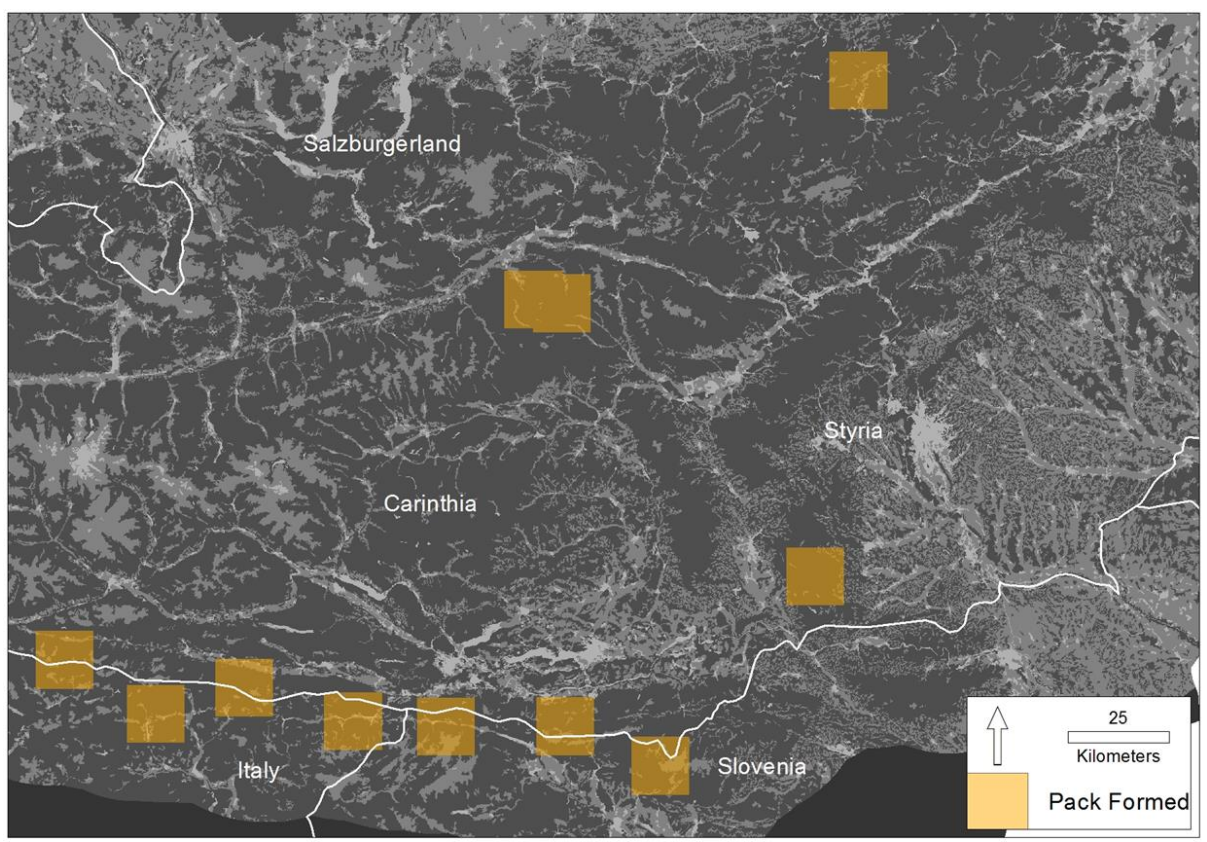

Figure 7: Locations of pack formation over the ten runs of $\$ 4$.

\section{Pack formation timeline}

The time step of pack formation for the four models was noted (Figure 8). S2 demonstrates early pack formations followed by less frequent pack formation in later time steps. S3 shows the opposite: despite the low number of total packs, most were formed in the second half of model runs. S4 shows a fairly uniform temporal distribution of pack formation.

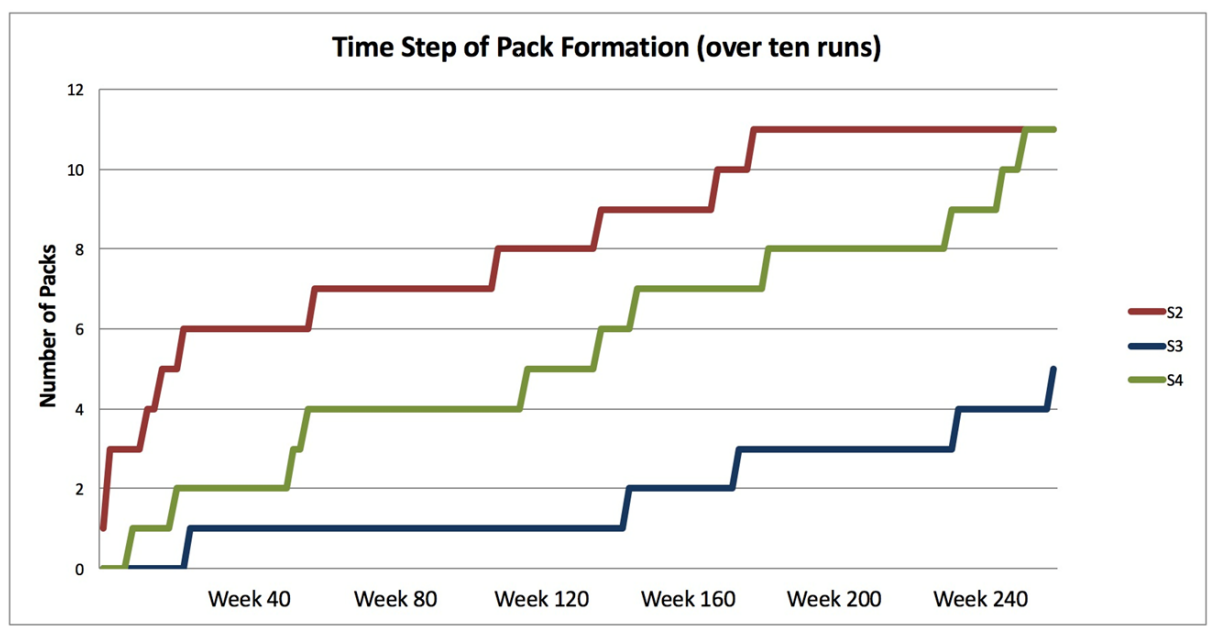

Figure 8: Time step of pack formation over the ten runs of each model. 


\section{Discussion and Conclusion}

The model developed here is a simple agent-based model used for exploring wolf recolonization in Austria; it uses literature-derived values related to wolf behaviour for model parameterization. Though not comprehensively validated, comparison of the model outputs with expert knowledge about recent populations of wolves in Austria indicates plausible correspondence to reality. Model run averages for the high likelihood model (S3) indicated an average of over 3 wolves alive in Austria at any given time step, while expert estimates were that there were usually around 5 wolves in the past five years. S3 also had an average total of 21.7 total wolves per model run compared to estimates of 17-18 over the last five years. It is apparent that minor changes to model parameters that are still within the realms of realistic values can result in outcomes even closer to estimates of reality. For instance, S4 (improved-attitudes scenario) resulted in an average of 5.38 wolves at any given time step.

This model does not purport to be an accurate prediction of where and when recolonization will occur. Rather, it is a learning tool that helps in describing the key components of the system and by demonstrating the mechanisms that will result in recolonization. Determining the time and place of pack formation is challenging, but the model has shown that in all but the most pessimistic conceptualizations of wolf movement and survival, a pack is likely to form. Of course, more data and improved understanding of the individual actions that make up each sub-model would result in a better model. For instance, wolf movement decisions are certainly more complicated than the habitat-dependent walk employed in the model. However, an attempt to model wolf movement truly accurately would require more empirical data on wolf movement as well as the integration of animal psychology literature. Any improvements in sub-model formulation would undoubtedly give the model more accuracy and power. As many such improvements are possible, the model described above should be seen as a framework that can be expanded upon and developed further to result in even better understanding of the system. This assertion parallels that of Watkins et al. (2014), who stated that in their ABM of Jaguar movements, framework parameters should be seen as a starting point in an iterative process that can be improved continually and compared with future empirical data.

According to this model, pack formation may be likely in Austria in the medium term. This sentiment was shared by an expert on wolves in Austria with whom we met, though he stressed the extreme uncertainty in predicting when and where pack formation may occur. While creating such a model may not point to precisely where and when pack formation will occur, it does help in identifying several things about the process that may be useful for management. First, the maps of likely dispersal locations and of known areas of pack formation give an idea of where likely areas for pack formation are (with admittedly high degrees of uncertainty). However, perhaps more importantly, these maps show specifically which areas are highly unlikely for pack formation. Secondly, the maps of wolf presence density may aid in identifying potential movement corridors, between source populations, which have attributes desirable for wolves and may be vital in fostering gene flow between the three distinct populations surrounding Austria. Finally, the ability to run different scenarios allows dynamic analysis of the phenomenon, allowing changes to parameter values 
reflecting changes in known empirical data (i.e. the model can be modified to reflect known wolf locations or Austria-specific mortality rates once these are established).

\section{Management}

These preliminary results can help in guiding management policy. In the conversations we had with residents of Carinthia, it was apparent that few people knew that any wolves were present in Austria, let alone what the implications of recolonization might be for them. Identifying likely areas (and ruling out unlikely ones) of wolf movement and pack formation can help management agencies in being proactive in outreach strategies, spreading information that prepares stakeholder groups who have more negative attitudes. For instance, management agencies could popularize husbandry practices that minimize conflict, stress the low levels of personal danger wolves pose to humans, and educate stakeholders about what to do in the case of conflict. Such outreach has been undertaken in Wiesbaden, Hesse, Germany, which has also had several transient wolves and similar near-term potential for pack formation (Klein, 2015). Here, proactive management has centred on the creation of a wolf management action plan that includes the creation of instructional handbooks for walkers in potential wolf areas, calls for two wolf management experts per county to handle conflict and outreach, training for farmers and shepherds in herd protection, and free access to electrified fences. Perhaps more importantly during the early stages of recolonization, establishing such a management plan attracts media coverage that prepares and educates the public. Such proactive outreach and management are costly, but having information on possible dispersal patterns can allow for their spatial prioritization.

\section{Lessons learned}

While general limitations in the application of this exploratory ABM are outlined in the discussion above, there are several specific technical areas in which improvements are needed to increase reliability. First, more rigorous calibration for each sub-model would be beneficial. While qualitative patterns based on the literature review were followed for the sub-models here, conducting quantitative calibration by either (a) collecting data locally as wolf populations expand or (b) using data from surrounding populations to derive parameters would allow for a vastly improved model. Along with this, sensitivity and uncertainty analysis that goes beyond assessing the four sets of literature-based scenarios should be conducted to better guide further model development. Due to the lack of field data and the associated difficulties in calibration, validation, sensitivity and uncertainty analysis, the overall uncertainty of the model was high.

There were also computational limitations introduced by using Agent Analyst. While this modelling environment had strong capabilities in organizing rule sets and observing model runs, it proved challenging to format visual model outputs (e.g. vector data would have been preferred to raster for presence locations and trajectories) and to automate multiple model runs to obtain a larger sample of outputs. 


\section{Conclusion}

Here, an exploratory agent-based model of wolf recolonization in Austria was demonstrated. Using literature-derived values for wolf behaviour, the model offered exploratory results that qualitatively reproduced system behaviour from the literature and resulted in wolf population estimates which were quite close to expert estimates for the region. This indicates that the exploratory $\mathrm{ABM}$ gave a plausible representation of the complex processes involved in the system at hand and should be thought of as a framework for further model refinement as more data and knowledge become available. Even with the challenges inherent in modelling a complex process with high levels of uncertainty, these results can foster further thought about the recolonization process by management agencies and the public alike.

\section{Acknowledgements}

This work was made possible through a generous scholarship from the Austrian Marshall Plan Foundation. The authors would also like to thank Dr Georg Rauer for discussing the current status of wolves in Austria with us.

\section{References}

Bankes, S., 1993. Exploratory modeling for policy analysis. Oper. Res. 41, 435-449.

Blanco, J.C., Cortés, Y., 2007. Dispersal patterns, social structure and mortality of wolves living in agricultural habitats in Spain. J. Zool. 273, 114-124.

Chapron, G., Arlettaz, R., 2006. Using models to manage carnivores. Science 314, 1682.

Ciucci, P., Boitani, L., Francisci, F., Andreoli, G., 1997. Home range, activity and movements of a wolf pack in central Italy. J. Zool. 243, 803-819.

Dungler, H., 2008. Frame conditions for a possible recovery of wolves in Austria, in: Perspectives of Wolves in Central Europe. Malenovice.

Enserink, M., Vogel, G., 2006. The carnivore comeback. Science 314, 746-749.

Grimm, V., Berger, U., DeAngelis, D.L., Polhill, J.G., Giske, J., Railsback, S.F., 2010. The ODD protocol: A review and first update. Ecol. Model. 221, 2760-2768.

Hurford, A., Hebblewhite, M., Lewis, M.A., 2006. A spatially explicit model for an Allee effect: Why wolves recolonize so slowly in Greater Yellowstone. Theor. Popul. Biol. 70, 244-254.

Jedrzejewski, W., Schmidt, K., Theuerkauf, J., Jedrzejewska, B., Okarma, H., 2001. Daily movements and territory use by radio-collared wolves (Canis lupus) in Bialowieza Primeval Forest in Poland. Can. J. Zool. 79, 1993-2004.

Johnston, K.M., North, M.J., Brown, D.G., 2013. Introducing agent-based modeling in the GIS environment, in: Agent Analyst: Agent-Based Modeling in ArcGIS. Redlands, CA: Esri Press, pp. $1-30$.

Klein, T., 2015. Wölfe auf Wanderschaft, HR-info: Environment. HR-info.

Macal, C.M., North, M.J., 2009. Agent-based modeling and simulation, in: Winter Simulation Conference, Austin, Texas. pp. 86-98.

Marucco, F., McIntire, E.J.B., 2010. Predicting spatio-temporal recolonization of large carnivore populations and livestock depredation risk: wolves in the Italian Alps. J. Appl. Ecol. 47, 789-798.

McLane, A.J., Semeniuk, C., McDermid, G.J., Marceau, D.J., 2011. The role of agent-based models in wildlife ecology and management. Ecol. Model. 222, 1544-1556. 
Mech, D.L., 1970. The Wolf: The Ecology and Behavior of an Endangered Species. Garden City, NY: Natural History Press.

Okarma, H., Jędrzejewski, W., Schmidt, K., and others, 1998. Home ranges of wolves in Białowieża Primeval Forest, Poland, compared with other Eurasian populations. J. Mammal. 79, 842-852.

Parker, D.C., Manson, S.M., Janssen, M.A., Hoffmann, M.J., Deadman, P., 2003. Multi-agent systems for the simulation of land-use and land-cover change: a review. Ann. Assoc. Am. Geogr. 93, 314337.

Pitt, W.C., Box, P.W., Knowlton, F.F., 2003. An individual-based model of canid populations: modelling territoriality and social structure. Ecol. Model. 166, 109-121.

Rabinowitz, A., Zeller, K.A., 2010. A range-wide model of landscape connectivity and conservation for the jaguar, Panthera onca. Biol. Conserv. 143, 939-945.

Railsback, S.F., Grimm, V., 2012. Agent-Based and Individual-Based Modeling: A Practical Introduction. Princeton, NJ: Princeton University Press.

Ripple, W., Beschta, R., 2003. Wolf reintroduction, predation risk, and cottonwood in Yellowstone National Park. Forest Ecology and Management 184, 299-313.

Salvatori, V., Linnell, J., 2005. Report on the conservation status and threats for wolf (Canis lupus) in Europe. Council of Europe.

Schafer, M., 2012. The National Wolf Strategy in Austria. Zurich: Swiss Federal Institute of Technology Zurich.

Watkins, A., Noble, J., Foster, R., Harmsen, B., Doncaster, C.P., 2014. Detailed behaviour ABM of jaguar movement. Figshare. http://dx.doi.org/10.6084/m9. figshare.1093834

Wiegand, T., Knauer, F., Kaczensky, P., Naves, J., 2004. Expansion of brown bears (Ursus arctos) into the eastern Alps: A spatially explicit population model. Biodivers. Conserv. 13, 79-114.

Zeiler, H., Zedrosser, A., Bath, A., 1999. Attitudes of Austrian hunters and Vienna residents toward bear and lynx in Austria. Ursus, 11, 193-200. 